\title{
Commentary
}

\section{Dicing Up MicroRNA Gene Expression Profiles in Normal and Neoplastic Smooth Muscle Cells}

\author{
Joseph M. Miano \\ From the Aab Cardiovascular Research Institute, University of \\ Rochester School of Medicine and Dentistry, Rochester, New York
}

Cellular identity and physiology are primarily a function of a cell's gene/protein expression profile, which are controlled transcriptionally by over 1400 transcription factors and post-transcriptionally through nearly 1000 microRNAs (miRs). Intergenic and intronic miRs located on the opposite strand of a host gene are transcribed through the same RNA polymerase II-dependent processes as those at protein-coding loci; however, miR genes undergo sequential cleavage through the type III ribonucleases Drosha and Dicer to generate mature, 21- to 24-nucleotide miRs. ${ }^{1}$ Once fully processed, the guide strand of the mature miR undergoes partial complementary base pairing with target sequences located in processed messenger RNAs to effect translational suppression or mRNA degradation. ${ }^{2}$ Significant interplay exists between transcription factors and miRs, with each class of regulators influencing expression and activity of the other to achieve homeostatic gene/protein expression profiles. ${ }^{3,4}$ Clearly, aberrations in this delicate balance of regulation have profound influence on the phenotype of a cell. For example, miRs are important determinants of an embryonic stem cell's ability to self-renew or differentiate, two opposing processes intricately tied to the development of neoplasia. ${ }^{5}$ Indeed, mounting data support altered miR expression in the cancer phenotype, with families of miRs acting as either tumor suppressors or oncogenes (oncomiRs). ${ }^{6}$ Expression profiling of both miR genes and protein-coding genes has thus resulted in subclassifications of tumor types and, by extension, new ideas about the origins and potential treatment of human neoplasias.

Smooth muscle cells (SMCs) found in blood vessels and visceral organs less frequently exhibit neoplastic transformation than epithelial cells. Ironically, however, SMCs display considerable phenotypic modulation in a variety of pathological conditions, including atherosclerosis, asthma, and obstructive bladder disease, where the normal program of contractile function is subverted to one of hypertrophy, proliferation, migration, and matrix hypersecretion. ${ }^{7}$ Among SMC lineages, uterine SMCs display perhaps the highest potential for neoplastic transformation, ranging from the more prevalent uterine leiomyomas (ULM) to the less frequent, though highly aggressive, malignant leiomyosarcomas (ULMS). ${ }^{8}$ All SMC lineages appear to gain their unique identity through the activity of myocardin and serum response factor, which together constitute a molecular switch for SMCrestricted cyto-contractile and regulatory gene expression via serum response factor-binding CArG boxes. ${ }^{9-14}$ Interestingly, serum response factor controls a growing number of miRs including the bicistronic miR$143 / 145$ gene. ${ }^{4,15,16}$ In a series of recent complementary studies, miR-143/145 was shown to coordinate aspects of the SMC differentiated state. ${ }^{4,16-19}$ However, the fact that miR-143/145 knockout mice display clear evidence of SMC differentiation implies that additional miRs play a role in conferring the complete SMC differentiation program. ${ }^{16,18,19}$

In this issue of The American Journal of Pathology, Danielson et al. ${ }^{20}$ report on novel miR profiles during the transition from bone marrow-derived mesenchymal stem cells (MSCs) to differentiated SMCs as well as in ULM and ULMS of human origin, and they provide novel insight into how miR profiling may be exploited to assist clinicians in accurately diagnosing these tumor types. The authors first established two in vitro models of MSCderived SMC differentiation, showing reciprocal regulation of such SMC differentiation markers as the gold standard marker for SMCs, MYH11, ${ }^{21}$ with the MSC markers CD73 and CD105. Importantly, an in vitro gel contraction assay demonstrated that induction of contractile markers correlates with functional activity. The authors then used siRNA studies to show that Dicer is necessary for the normal expression of key SMC contractile markers during the transition from bone marrow MSCs to differentiated SMCs. These results are consistent with a recent

Accepted for publication May 18, 2010

CME disclosure: The author did not disclose any relevant financial relationships.

Address reprint requests to Joseph M. Miano, Ph.D., Aab Cardiovascular Research Institute, University of Rochester School of Medicine and Dentistry, 601 Elmwood Avenue, Box CVRI, Rochester, NY 14642. E-mail: j.m.miano@rochester.edu. 
report from Albinsson et $\mathrm{al}^{22}$ showing attenuated SMC contractile marker expression and altered actin cytoskeletal dynamics in SMCs where Dicer was deleted specifically in SMC lineages. Although Danielson et al did not report on the contractile activity of SMCs with reduced levels of Dicer, Albinsson et al ${ }^{22}$ showed clear evidence of defective agonist-induced contractile activity in vessels lacking normal levels of Dicer. Thus, there is now unambiguous evidence supporting a critical role for normal miR expression in mediating the biochemically and functionally differentiated state of SMCs.

Having established a role for Dicer in the SMC differentiated phenotype, Danielson et al went on to perform microarray screening for specific miR expression profiles in their models of in vitro SMC differentiation. Remarkably, both models of SMC differentiation displayed similar patterns of miR expression, suggesting that the transition of human MSCs into differentiated SMCs involves a specific subset of miR genes. In particular, let7b showed increases in expression, whereas miR-93, miR-106a, and miR-20a displayed reduced expression during the transition from MSC to SMC. Interestingly, let7 family members were recently reported to limit mouse embryonic stem cell renewal in part through the inhibition of cell cycle-associated miRs, which might include miR-93, miR106a, and miR-20a. ${ }^{5}$ This implies that there are miR genes that promote a "stemness" phenotype and those that oppose self-renewal, thus allowing for specific programs of cell differentiation to proceed.

It is curious that the SMC-specific miR-143/145 was not listed in their normal SMC profile. Although miR-143/145 can direct SMC contractile gene expression, their absence does not negate SMC differentiation in vivo. ${ }^{16,18,19}$ Thus, the lack of significant differences in miR-143/145 expression in the in vitro stem cell models of SMC differentiation studied here may not be entirely surprising. In fact, a recent report revealed a critical role for miR-10a in directing mouse embryonic stem cells to differentiate into SMCs, suggesting that there are more miR genes to uncover that contribute to the fully differentiated SMC phenotype. ${ }^{23}$

Next, the authors performed microarray screening for miRs in samples of normal myometrium, ULM, and ULMS followed by hierarchical clustering to ascertain similarities and differences between these tumor types. This elegant analysis revealed that ULM and ULMS could be segregated based on their miR expression profile. Of major importance, bioinformatic analysis further showed that whereas the miR expression profile of ULM more closely resembled normal myometrium, the ULMS profile appeared similar to that of hMSCs, which might suggest that there is a continuum of phenotypes between either bone marrow-derived stem cells that home to the uterus or resident stem cells within the uterus and the cells comprising ULMS. In this context, expression levels of some let7 family members, which are inhibitory for stem cell renewal, ${ }^{5}$ were reduced in ULMS. The latter would further imply that cells of the ULMS tumor type are less differentiated. An important and unresolved question is whether ULMS arises from resident stem cells within the myometrium or from normal myometrial SMCs that undergo de-differentiation.
Danielson et al used sophisticated bioinformatic analysis applied over a distance timeline of SMC differentiation to show that while ULM samples clustered with normal myometrium late on the time scale, ULMS exhibited a broader distribution, perhaps reflecting individual heterogeneity in phenotype between ULMS samples. Despite such scatter in ULMS phenotypes, ULM and ULMS did share in expression levels of some miRs, most notably miR-21, which was found to be elevated in both ULM and ULMS, consistent with a previous report of miR expression in cultured SMCs from ULM. ${ }^{24}$ Though there may be a few commonalities in miR expression between ULM and ULMS, Danielson et al correctly noted that these results do not necessarily imply evolution of ULMS from ULM, nor do they provide insight into whether these tumor types arise from a common precursor. As a matter of fact, the results offered by Danielson et al show only six differentially expressed miRs in ULM as compared with 50 in ULMS. In this context, ULMS showed decreases in miR-143/145 with apparently no changes of this bicistronic miR in ULM samples. These results are somewhat at odds with previous data demonstrating elevated miR145 in cultured SMCs derived from ULM tumors. ${ }^{24}$ Different experimental conditions or sample heterogeneity likely explain these contrasting observations.

Nearly one-third of the miRs interrogated by Danielson et al showed similar patterns of expression in ULMS and normally differentiated SMCs. This indicates that there may be shared features (transcriptional and post-transcriptional) between cells from ULMS and normal SMCs with common miRs directing processes associated with both the malignant and normal SMC differentiated program. Formal proof of this intriguing concept awaits further study of the mRNA transcript and protein profile of these samples.

The new results from Danielson et al, although limited in terms of sample size ( $n=10$ for each tumor type), provide novel insight into the molecular profile of SMC differentiation from a developmental and pathological perspective and arm the pathologist with new biomarkers that could be useful in classifying uterine tumor types. There are additional tumor types in the uterus ${ }^{8}$ whose molecular profiling would be of interest to compare with those described here. In addition, it will be important to try and link the miR expression profiles described in the report of Danielson et $\mathrm{al}^{20}$ to bona fide SMC-specific cyto-contractile markers (MYH11, ACTG2, CNN1, etc) as well as other histological features (eg, cell/nuclear morphology and mitotic index) that define tumor grade. Further, it would be informative here to know what the level of myocardin expression is across ULM and ULMS samples since this master regulator of SMC differentiation has been reported to be highly expressed in human ULMS and retroperitoneal LMS, ${ }^{25,26}$ but reduced in other cases of human ULMS. ${ }^{27}$ These disparate findings likely highlight the heterogeneity in tumor phenotypes, which Danielson et al clearly show for ULMS in their differentiation timeline study. Finally, it will be instructive to elucidate the target mRNAs for each miR expression profile defined in this and other reports. Such a task will require integrating the next generation of algorithms that reliably 
predict miR target sequences with transcriptomic and proteomic profiles in ULM versus ULMS samples. The definition of unique miR codes of gene expression distinguishing two uterine tumor types as reported by Danielson et al represents a critical starting point toward addressing these and other important questions pertaining to uterine tumor pathobiology.

\section{References}

1. Carmell MA, Hannon GJ: RNase III enzymes and the initiation of gene silencing. Nat Struct Mol Biol 2004, 11:214-218

2. Pratt AJ, MacRae IJ: The RNA-induced silencing complex: a versatile gene-silencing machine. J Biol Chem 2009, 284:17897-17901

3. Xu N, Papagiannakopoulos T, Pan G, Thomson JA, Kosik KS: MicroRNA-145 regulates OCT4, SOX2, and KLF4 and represses pluripotency in human embryonic stem cells. Cell 2009, 137:647-658

4. Cordes KR, Sheehy NT, White MP, Berry EC, Morton SU, Muth AN, Lee TH, Miano JM, Ivey KN, Srivastava D: miR-145 and miR-143 regulate smooth muscle cell fate and plasticity. Nature 2009, 460:705-710

5. Melton C, Judson RL, Blelloch R: Opposing microRNA families regulate self-renewal in mouse embryonic stem cells. Nature 2010, 463:621-626

6. Iorio MV, Croce CM: MicroRNAs in cancer: small molecules with a huge impact. J Clin Oncol 2009, 27:5848-5856

7. Owens GK, Kumar MS, Wamhoff BR: Molecular regulation of vascular smooth muscle cell differentiation in development and disease. Physiol Rev 2004, 84:767-801

8. D'Angelo E, Prat J: Uterine sarcomas: a review. Gynecol Oncol 2010, 116:131-139

9. Wang D-Z, Chang PS, Wang Z, Sutherland L, Richardson JA, Small E, Krieg PA, Olson EN: Activation of cardiac gene expression by myocardin, a transcriptional cofactor for serum response factor. Cell 2001, 105:851-862

10. Chen J, Kitchen CM, Streb JW, Miano JM: Myocardin: a component of a molecular switch for smooth muscle differentiation. J Mol Cell Cardiol 2002, 34:1345-1356

11. Miano JM: Serum response factor: toggling between disparate programs of gene expression. J Mol Cell Cardiol 2003, 35:577-593

12. Pipes GCT, Creemers EE, Olson EN: The myocardin family of transcriptional coactivators: versatile regulators of cell growth, migration, and myogenesis. Genes Dev 2006, 20:1545-1556

13. Parmacek MS: Myocardin-related transcription factors: critical coactivators regulating cardiovascular development and adaptation. Circ Res 2007, 100:633-644
14. McDonald OG, Owens GK: Programming smooth muscle plasticity with chromatin dynamics. Circ Res 2007, 100:1428-1441

15. Niu Z, Li A, Zhang SX, Schwartz RJ: Serum response factor micromanaging cardiogenesis. Curr Opin Cell Biol 2007, 19:618-627

16. Xin M, Small EM, Sutherland LB, Qi X, McAnally J, Plato CF, Richardson JA, Bassel-Duby R, Olson EN: MicroRNAs miR-143 and miR-145 modulate cytoskeletal dynamics and responsiveness of smooth muscle cells to injury. Genes Dev 2009, 23:2166-2178

17. Cheng Y, Liu X, Yang J, Lin Y, Xu DZ, Lu Q, Deitch EA, Huo Y, Delphin ES, Zhang C: MicroRNA-145, a novel smooth muscle cell phenotypic marker and modulator, controls vascular neointimal lesion formation. Circ Res 2009, 105:158-166

18. Boettger T, Beetz N, Kostin S, Schneider J, Kruger M, Hein L, Braun $\mathrm{T}$ : Acquisition of the contractile phenotype by murine arterial smooth muscle cells depends on the Mir143/145 gene cluster. J Clin Invest 2009, 119:2634-2647

19. Elia L, Quintavalle M, Zhang J, Contu R, Cossu L, Latronico MV, Peterson KL, Indolfi C, Catalucci D, Chen J, Courtneidge SA, Condorelli G: The knockout of miR-143 and -145 alters smooth muscle cell maintenance and vascular homeostasis in mice: correlates with human disease. Cell Death Differ 2009, 16:1590-1598

20. Danielson LS, Menendez S, Stephan-Otto Attolini C, Guijarro MV, Bisogna M, Wei J, Socci ND, Levine DA, Michor F, Hernando E: A differentiation-based miRNA signature identifies leiomyosarcoma as a mesenchymal stem cell-related malignancy. Am J Pathol 2010 , 177:908-917

21. Miano JM, Cserjesi P, Ligon KL, Periasamy M, Olson EN: Smooth muscle myosin heavy chain marks exclusively the smooth muscle lineage during mouse embryogenesis. Circ Res 1994, 75:803-812

22. Albinsson S, Suarez Y, Skoura A, Offermanns S, Miano JM, Sessa WC: MicroRNAs are necessary for vascular smooth muscle growth, differentiation, and function. Arterioscler Thromb Vasc Biol 2010, 30:1118-1126

23. Huang $H$, Xie $C$, Sun $X$, Ritchie RP, Zhang J, Chen YE: miR-10a contributes to retinoid acid-induced smooth muscle cell differentiation. J Biol Chem 2010, 285:9383-9389

24. Wang T, Zhang X, Obijuru L, Laser J, Aris V, Lee P, Mittal K, Soteropoulos $P$, Wei JJ: A micro-RNA signature associated with race, tumor size, and target gene activity in human uterine leiomyomas. Genes Chromosomes Cancer 2007, 46:336-347

25. Liang J, Wu L, Xiao H, Li N, Wang H, Cheng C, Bai R, Zhao Y, Zheng $\mathrm{H}$ : Use of myocardin in the classification of mesenchymal tumors of the uterus. Int J Gynecol Pathol 2010, 29:55-62

26. Perot G, Derre J, Coindre JM, Tirode F, Lucchesi C, Mariani O, Gibault L, Guillou L, Terrier P, Aurias A: Strong smooth muscle differentiation is dependent on myocardin gene amplification in most human retroperitoneal leiomyosarcomas. Cancer Res 2009, 69:2269-2278

27. Kimura Y, Morita T, Hayashi K, Miki T, Sobue K: Myocardin functions as an effective inducer of growth arrest and differentiation in human uterine leiomyosarcoma cells. Cancer Res 2010, 70:501-511 